\title{
Un campo que se mira a sí mismo. Acuerdos y desacuerdos en las reflexiones sobre el estatuto epistemológico de la Comunicación
}

\author{
A field that watches itself. Agreements and \\ discords about epistemological statute of \\ Communication
}

MARTA Rizo García

\section{Resumen}

La pregunta por la cientificidad de la comunicación no es nueva y ha dado lugar a múltiples posicionamientos, reflexiones y propuestas. La comunicación es un objeto de estudio que interesa a varias disciplinas, fundamentalmente sociales; sobre ello parece haber consenso. Tampoco está a discusión que el pensamiento comunicacional tiene una historia propia que ha dado lugar a producción académica en torno a los fenómenos comunicacionales. Y más aún, que la comunicación debe necesariamente interactuar con otros campos de conocimiento en su quehacer científico, tampoco es algo que pueda someterse a debate. Sin embargo, no existe consenso en torno a la naturaleza científica de la comunicación. Desde las visiones que la consideran únicamente como un objeto de estudio susceptible de ser mirado por disciplinas muy diversas, hasta aquellas que abogan por la comprensión de la comunicación como un campo transdisciplinario, pasando por aquellas otras que la comprenden como una disciplina particular, existen muchas miradas en torno al estatuto epistemológico de las comúnmente llamadas, en plural, Ciencias de la Comunicación. En estas páginas se recogen algunas de estas visiones, con miras a ofrecer una suerte de estado de la cuestión en torno al tema objeto del debate: el estatuto epistemológico de la comunicación. Dada la imposibilidad de 
dar cuenta de la totalidad de posicionamientos en torno al tema, en este texto se destacan únicamente algunos de ellos, fundamentalmente procedentes del espacio iberoamericano.

Palabras clave: campo académico, epistemología, ciencia, disciplina, interdisciplina.

\begin{abstract}
The question about scientific status of communication isn't new and has given rise multiple positions, reflections and proposals. Communication is an object of study that interests to several disciplines, fundamentally social; it seems to have consensus about this question. It isn't either to discussion that communicational thought has an own history that has given a lot of academic production around communicational phenomena. And still more, that Communication must necessarily interact with other knowledge fields in its scientific task can't be put under debate. Nevertheless, consensus around the scientific status of communication doesn't exist. There are visions that consider communication as an object of study watched from very different disciplines; proposals that understand Communication like a transdisciplinary field; others that understands Communication like a particular discipline. Really, there are many positions around the epistemological statute of commonly called Communication Sciences. These pages present some these visions, in order to offering a state of the question about the epistemological and scientific statute of communication. It's impossible to review the totality of positions about the question; therefore, in this text only stand out some of these, specially coming from Latin American region.
\end{abstract}

KeYwords: academic field, epistemology, science, discipline, interdisciplinary.

\title{
La naturaleza (problemática) del debate en torno al estatuto epistemológico de la comunicación.
}

La pregunta por la cientificidad de la comunicación no es nueva y ha dado lugar a múltiples posicionamientos, reflexiones y propuestas. En muchos casos, se trata de planteamientos muy distantes entre sí, incluso antagónicos; en otros más, existen puntos de convergencia entre varios de los investigadores preocupados por el debate en torno al estatuto epistemológico de la comunicación. En este sentido, y siguiendo a León Duarte, "las cuestiones centrales que patentiza el campo académico de la comunicación, y los desafíos que en la actualidad se le presentan a su investigación, tienen que ver fundamentalmente con su estatuto disciplinario" (León, 2009). 
Que la comunicación es un objeto de estudio que interesa a varias disciplinas, fundamentalmente sociales, es algo que poco puede ponerse en duda. Que el pensamiento comunicacional tiene una historia propia que ha dado lugar a producción académica en torno a los fenómenos comunicacionales, tampoco está a discusión. Y más aún, que la comunicación, como espacio académico, debe necesariamente interactuar con otros campos de conocimiento en su quehacer científico, tampoco es algo que pueda someterse a debate. Sin embargo, y aquí está el punto nodal, no existe consenso en torno a la naturaleza científica de la comunicación. Desde las visiones que la consideran únicamente como un objeto de estudio susceptible de ser mirado por disciplinas muy diversas, hasta aquellas que abogan por la comprensión de la comunicación como un campo transdisciplinario, pasando por aquellas otras que la comprenden como una disciplina particular, existen muchas miradas en torno al estatuto epistemológico de las comúnmente llamadas, en plural, Ciencias de la Comunicación.

En estas páginas se recogen algunas de estas visiones, con miras a ofrecer una suerte de estado de la cuestión en torno al tema objeto del debate: el estatuto epistemológico de la comunicación. Obviamente, existen limitaciones para dar cuenta de la totalidad de posicionamientos en torno al tema. De ahí que en este texto se destaquen únicamente algunos de ellos, fundamentalmente procedentes del espacio iberoamericano.

La comunicación es coextensiva a la sociedad: "la sociedad, en cualquiera de sus escalas, es impensable sin la comunicación. Y bajo este aspecto, ella interesa directa o indirectamente, explícita o implícitamente, a todas las disciplinas sociales" (Giménez, 2009: 1). De ahí que pueda comprenderse a la comunicación como un "hecho social total" (Mauss, 1979), porque remite de un modo u otro a todas las instancias de la sociedad.

Sin embargo, y casi de forma paradójica, las Ciencias de la Comunicación reivindican sus fronteras, su objeto propio, sus tradiciones y sus esquemas explicativos. Según Giménez (2009: 1), "esta situación paradójica se explica por el carácter híbrido de la disciplina y por su vocación natural a la pluralización". De ahí que los debates sobre el estatuto de la comunicación sean interminables. Hasta cierto punto, entonces, es natural que aún y con una historia de cerca de un siglo, el campo de la comunicación siga preguntándose acerca de su especificidad como campo de construcción de conocimiento.

Nicolás Sarale es muy claro al afirmar lo siguiente: "No decimos nada nuevo al enunciar que el campo académico de la comunicación se caracteriza por su indeterminación y su complejidad en cuanto a la definición de su objeto teórico propio y a su constitución disciplinaria" (Sarale, 2008:1). El autor ofrece un mapa de las tendencias de opinión, y señala a autores como Luiz C. Martino (2002), que pone énfasis en la polisemia del término comunicación; Guillermo Orozco (1994), que habla de la triple dimensión de la comunicación -"como proceso humano fundamental, como disciplina de conocimiento y disciplina - en-práctica o profesión"-; Raúl Fuentes (1999), para quien "el estatuto disciplinario de los estudios sobre la comunicación es, quizá, el tema crucial del debate sobre el pasado, el presente y, sobre todo el futuro de nuestro campo académico"; y Roberto Follari (2003), 
que critica la idea de interdisciplinariedad, pues entiende que pensar que cualquier tema puede ser objeto de los estudios comunicológicos, resta a éstos la posibilidad de cualquier identidad precisable. El panorama que ofrece la revisión de Sarale (2008) da cuenta de la multiplicidad de posturas que se apuntó anteriormente.

Es entonces propósito del presente ensayo la exposición de algunas de estas posturas, no sólo con la finalidad de ofrecer un mapa de tendencias, interesante por sí mismo, sino también de discutir los alcances y limitaciones de la discusión en la que éstas se enmarcan.

En este sentido, interesa no tanto imponer un único modo de ver, sino plantear las múltiples propuestas que existen en torno a la cientificidad de la comunicación como campo de construcción de conocimiento. El debate no está exento de conflictos, pues como afirma Karam,

\footnotetext{
la pregunta por la cientificidad de la comunicación es con frecuencia molesta para algunos sectores y grupos (dentro y fuera de la comunicación), ya que parece pretenciosa y remite a un debate en el cual, se supone, quienes enuncian la pregunta pertenecen a un grupo que excluye a quienes la pueden responder negativamente (...) más allá de la respuesta, lo importante es el debate y la discusión que puede tener muy diversas áreas de aplicación que van desde la formación de currículos, hasta proyectos científicos que den una mayor fundamentación a la reflexión sobre los objetos y métodos de la comunicación (Karam, 2007: 98).
}

Si bien es notoria la falta de consenso en torno al estatuto disciplinario de la comunicación, parece no existir este problema, o al menos no en la misma magnitud, a la hora de discutir cuál es el objeto de estudio de la comunicación. Desde hace varias décadas, el canónico objeto de estudio "medios" ha sido desplazado por otro tipo de procesos de mucha mayor escala. Este desplazamiento supone, creemos, un logro importante para construir consensos al interior del campo de la comunicación.

Dicho en otros términos, después del predominio de las escuelas de corte lineal o procesual que asociaban la comunicación casi exclusivamente con la transmisión de mensajes, hoy en dia tiene más aceptación una concepción de la comunicación asociada a la construcción de significados. Para Luiz Martino (2002), por ejemplo, el "objeto de estudio de la comunicación son las prácticas comunicacionales liberadas por la sociedad compleja y hasta entonces desconocidas en otros tipos de sociedades históricas" (citado en Olmedo, 2012: 7). El énfasis no se encuentra en el mero intercambio informativo, sino que va mucho más allá y toma en cuenta, primordialmente, los procesos de producción de sentido (Fuentes, 2003).

En torno a estos consensos - sobre el objeto de estudio de la comunicación - y disensos - acerca de la especificidad científica del campo - versan las siguientes páginas. Toda vez que no existen visiones cerradas, autónomas unas de otras, no se considera conveniente organizar el ensayo en apartados que reflejen, en su interior, posturas 
específicas y absolutamente claras. De ahí que se prefiera una organización por lo que podemos denominar tendencias de pensamiento. Tendencias que, como se verá, no son unívocas ni lineales.

Coincidimos con Martino cuando afirma que "entre el rango de ciencia constituida o sólo un campo de intersección de saberes, el estatuto de la comunicación social ha variado y dividido opiniones a lo largo de las décadas" (Martino, 2002: 75). Sin pretender recoger todas estas opiniones, empresa inabarcable en un solo documento, proponemos algunas trazas que permiten identificar el estado de la discusión en torno al estatuto científico y epistemológico de la comunicación.

La pertinencia de abordar esta cuestión es clara pero vale la pena recordarla: saber qué hemos hecho como campo y conocer cómo nos vemos a nosotros mismos como campo de conocimiento, permitirá no sólo una mayor claridad en el avance del mismo, sino que también ayudará a consolidar lo que en su seno se produce. Como afirma Martín Serrano, "una teoría llega a ser referencia científica para un campo del saber después de que se haya tomado a sí misma como objeto de conocimiento" (Martín Serrano, 1986: 17). Aunque en este caso el objeto no es la teoría de la comunicación en sí misma, parece importante rescatar esta idea, estableciendo un paralelismo entre la teoría y el campo, para poder afirmar con absoluto convencimiento que un campo de conocimiento puede llegar a ser referencia científica (sea o no ciencia o disciplina particular, como veremos), sólo si se toma a sí mismo como objeto de conocimiento.

\section{La comunicación: fenómeno, objeto y campo de estudios.}

"El tener la comunicación un objeto tan difuso y permanente, tan esquivo y complejo, hace muy difícil delimitarlo como condicionante para su carácter científico" (Karam, 2007: 102). El objeto de estudio de las ciencias de la comunicación es algo que, en sí mismo, conlleva al debate, a encuentros y desencuentros. Sin embargo, como se comentó anteriormente, parece existir un consenso bastante notorio en torno al tránsito de los medios a las mediaciones o procesos comunicativos que trascienden (incluyéndolos pero no reduciéndose a ellos) a los medios de difusión.

Los fenómenos comunicativos son hechos sociales interesantes para cualquier disciplina relacionada con la sociedad humana y con el comportamiento humano. 0 dicho de otra forma, "la comunicación es atravesada por todos los órdenes de lo humano, y también ella los atraviesa" (Sierra, 2005: 93). De ahí que la comunicación sea un objeto de estudio compartido por varias miradas disciplinarias y campos de saber.

Desde este punto de vista, la comunicación, parafraseando a Enrique Sánchez Ruiz (1997), no tiene ni ha tenido un campo disciplinar propio, sino un dominio de estudio, más o menos común, alrededor del cual se ha conformado el campo sociocultural. Este 
dominio, a decir del autor, "ha sido, es y quizá tendrá que seguir siendo una encrucijada inter y transdisciplinaria dentro de las ciencias sociales y humanas" (Sánchez Ruiz, 1997: 57).

Lo anterior puede relacionarse con la idea de la autonomía relativa de la comunicación que propone Hercovici (1998). Para el autor, "cuando la dimensión intrínsecamente histórica y social de la comunicación es afirmada, no es posible autonomizar el objeto de estudio" (Hercovici, 1998: 140). Necesariamente, entonces, la comunicación -como espacio académico - comparte el objeto de estudio con otros campos disciplinarios, generalmente asociados con las ciencias sociales y las humanidades, aunque no reducidos a éstas.

En una línea similar se sitúan las reflexiones de Erik Torrico, para quien el objeto de la comunicación es "el proceso social de producción, circulación mediada, intercambio desigual, intelección y uso de significaciones y sentidos culturalmente situados, que es algo de naturaleza socialmente estructural (constitutivo) e inseparable -para fines teóricos e investigativos - de las otras dimensiones analíticas de la vida social" (Torrico, 2004: 21). Las otras dimensiones analíticas de la vida social refieren a lo económico, lo político, lo cultural, etc., ámbitos que sin duda no pueden obviarse en las reflexiones en torno a los fenómenos comunicativos propios de las sociedades actuales.

El contexto del nacimiento de la comunicación como campo de saber fue sobre todo pragmático; es decir, en los inicios, la orientación de la comunicación fue fundamentalmente empirista y práctica. Con el paso de los años, la diversificación de las orientaciones presentes en el conjunto de aportaciones que se hacen desde el espacio académico de la comunicación ha dado lugar, según ciertas posturas, a una "fragmentación anárquica" (Giménez, 2009: 4). En este escenario, no es entonces extraño que existan posturas que asuman que las ciencias de la comunicación son "un campo emergente de la modernidad que aún no logra encontrar su sitio en el concierto científico de la transmodernidad" (Cornejo, 2007: 2). Idea que, sin embargo, no debe hacernos creer que la comunicación es únicamente un objeto transversal a muchos campos de conocimiento $y_{\text {, en ningún }}$ caso y bajo ninguna circunstancia, un espacio académico contenedor de ciertas formas de mirar la sociedad.

Si sobre algo existe consenso en el escenario actual de la comunicación, es que ésta se ha constituido más como un campo que como una ciencia o disciplina específica. Al respecto, María Immacolata Vassallo (2002) aplica el concepto de campo de Pierre Bourdieu ${ }^{1}$ a la comunicación, y sostiene que

el campo académico de la comunicación está constituido por: un conjunto de instituciones de educación superior destinadas al estudio y a la enseñanza de la comunicación, donde se produce la teoria, la investigación y la formación universitaria de los profesionales de la

\footnotetext{
1 Bourdieu define al campo como "un espacio social estructurado, un campo de fuerzas -hay dominantes y dominados, hay relaciones constantes, permanentes, de desigualdad, que se ejercen al interior de ese espacio - que es también un campo de luchas para transformar o conservar este campo de fuerzas" (Bourdieu, 1997: 48-49). En el campo se construye una visión interpretativa y de apuesta por la construcción de sentido. Y el campo promueve la existencia de objetos, discursos, sujetos, conocimientos y acciones.
} 
comunicación. Lo que implica que en ese campo se pueden identificar varios subcampos: el científico, implicado en prácticas de producción del conocimiento: la investigación académica tiene la finalidad de producir conocimiento teórico y aplicado por medio dela construcción de objetos, metodologías y teorias; el educativo, que se define por prácticas de reproducción de ese conocimiento, es decir, mediante la enseñanza universitaria de materias relacionadas con la comunicación, y el profesional, caracterizado por prácticas de aplicación del conocimiento y que promueve vínculos variados con el mercado de trabajo (Vassallo, 2002: 4).

En este punto aparece la triple condición de la comunicación que anteriormente mencionamos al referirnos a lo propuesto por Guillermo Orozco (1994). La comunicación, así entonces, es simultáneamente un campo educativo, profesional y científico o académico, además de ser, antes que cualquier otra cosa, un fenómeno sociocultural que todo ser humano experimenta cotidianamente y sobre el cual pueden afirmarse muchas cosas.

Cercano al concepto de campo académico, cuya legitimidad como término referido al espacio de producción de conocimiento sobre la comunicación pocos pueden poner en duda, Erick Torrico habla de la comunicación como "cultura académica", entendida como el conjunto de "elementos básicos que pueden ser catalogados como producto y síntesis de una trayectoria de depuración y acumulación teórica que sí existen desde el punto de vista comunicológico" (Torrico, 2005: 41). El autor comprende la comunicología como "la mirada analítica especializada que aborda la dimensión comunicacional de los procesos sociales en interacción con otras que sean pertinentes a los objetos de estudio concretos que se investigue" (Torrico, 2005: 41). De ahí que su visión se acerque más a entender la comunicación como un campo interdisciplinario.

Torrico retoma el concepto de cultura académica de Wallerstein (1999: 14), en el sentido de "conjunto de premisas que la mayoría de los miembros de la comunidad científica comparte subconscientemente y que no están sujetas a debate sino rara vez". Afirma Torrico que la trayectoria del pensamiento comunicacional, lo producido en el seno del campo académico de la comunicación, permite identificar algunos aspectos que, a decir del autor, son compartidos por gran parte de los investigadores que se adscriben a dicho campo de conocimiento. Por ejemplo, existe cierto consenso, como ya se mencionó, en torno a la superación de la asociación de la comunicación con los medios, lo cual lleva a afirmar que la comunicación debe ser comprendida, antes que cualquier otra cosa, como un "proceso social de producción, circulación, intercambio desigual y uso de significaciones y sentidos culturalmente situados y mediados o no por tecnologías" (Torrico, 2005: 45). El mismo autor considera que existen ciertas proposiciones generales sobre la comunicación que pocos pondrían en duda, a saber: 1) La comunicación es un proceso humano y social de interacción significante que opera mediante representaciones; 2) Sus elementos esenciales son los sujetos participantes, los lenguajes y los recursos de mediación que ellos emplean, las representaciones que construyen y el contexto social menor 
o mayor en que el proceso tiene lugar; 3) La comunicación está basada en convenciones socioculturales que interrelacionan agentes con estructuras y son capaces de expresarlos; 4) La comunicación puede posibilitar entendimientos mínimos o expandidos; 5) La comunicación contribuye a generar determinadas consecuencias perceptuales, cognitivas, afectivas y/o conductuales entre sus participantes; 6) La comunicación está vinculada al empleo de recursos tecnológicos que la mediatizan y pueden amplificar su alcance; y 7) La comunicación, siendo transversal a ellos, interactúa con otros procesos de la sociedad, en particular la cultura, la política y el desarrollo (Torrico, 2005: 45-46).

Según Torrico, "a esta altura del tiempo no cabe duda alguna respecto a la posibilidad de demarcar, como ya se indicó, la mirada comunicológica. Lo que resta es completar los aspectos que la legitimen en el ámbito de la observación científica" (Torrico, 2005: 47). Si bien podemos compartir hasta cierto punto que, aunque existen elementos compartidos por la comunidad de investigadores, es difícil hablar de una ciencia comunicológica demarcada de otras disciplinas y completamente autónoma, este planteamiento hace emerger algunas interrogantes que aún no están resueltas: ¿Cómo se pueden completar estos aspectos de los que habla Torrico? ¿Existe el consenso suficiente más allá de las proposiciones generales sobre el fenómeno comunicativo? ¿La dispersión en cuanto a enfoques teóricos puede ser vista como un obstáculo para tal propósito?

\section{Propuestas interdisciplinarias y transdisciplinarias para pensar el campo de la comunicación}

Gran parte de las discusiones en torno al estatuto epistemológico y científico de la comunicación derivan de apreciaciones en torno a la necesidad de consolidar el campo como una disciplina autónoma, en un extremo, y como un espacio transdisciplinario, en el otro. En este apartado se presentan algunas de las ideas que emergen de estas discusiones en torno a la naturaleza disciplinaria, interdisciplinaria o transdisciplinaria de la comunicación. En este punto, cabe mencionar que el problema u objeto de discusión no es tanto la comunicación como fenómeno social que puede convertirse en un objeto de estudio de diversas disciplinas; como objeto, por tanto, la comunicación es sin duda interdisciplinaria. Sin embargo, no existe un consenso similar en cuanto a la naturaleza del campo de conocimiento que, para bien y para mal, comparte nombre con su objeto de estudio: la comunicación.

Según Mathei Dogan y Robert Phare (1991), el ciclo vital de las disciplinas sociales tiene cuatro fases: fundacional (se forma un patrimonio científico originario, es el momento de anclaje y de formación de un núcleo disciplinario inicial), de expansión (se enriquece el patrimonio inicial con la contribución de numerosos científicos), de especialización (se fragmentan las disciplinas en numerosas subdisciplinas) y de hibridación o amalgama 
(recombinación de especialidades o de fragmentos de disciplinas vecinas). (Citado en Giménez, 2012: 250). Para Giménez, el de la comunicación es un campo que se encuentra en esta última fase: la hibridación o amalgama. Para el autor, "la hibridación o amalgama consiste en la fusión, recombinación o cruzamiento de especialidades o fragmentos de disciplinas vecinas. No abarcan a disciplinas completas, sino sólo a sectores parciales de las mismas. No debe confundirse con 'multidisciplinariedad' o 'pluridisciplinariedad', que evoca la mera convergencia de monodisciplinas en torno a un mismo objeto de estudio, pero que conserva para cada cual celosamente sus presuntas fronteras" (Giménez, 2012: 250). Nos parce pertinente esta apreciación, dado que efectivamente, o al menos así parece verse en los hechos, lo producido a lo largo de la historia del pensamiento comunicacional es producto de la fusión de aportaciones disciplinarias muy diversas. De ello dan cuenta estudios que ponen énfasis, por citar sólo algunos ejemplos, en la relación entre procesos comunicativos e identidades culturales, la relación entre comunicación y procesos políticos y electorales, y los monopolios de las industrias de la comunicación, áreas temáticas que acercan al campo de la comunicación con la antropología, la ciencia política y la economía, respectivamente.

La epistemología contemporánea ha roto en gran medida el molde de las disciplinas científicas; o al menos, no parte de concebir a las disciplinas tal y como fueron pensadas en la modernidad occidental. De ahí que la noción de amalgama o hibridación que recupera Giménez nos parezca acertada. En un sentido similar, Acosta habla de la comunicación como una bisagra, y afirma que como objeto de estudio, la comunicación puede comprenderse como "un hilo rearticulador de intereses y estudios antes separados y que pueden ayudar a comprender la complejidad constitutiva de los procesos físicos, biológicos y antroposociológicos" (Acosta, 2010: 1).

Resulta problemático afirmar, según Acosta, "la existencia de 'una' teoría de la comunicación como disciplina autónoma y reconocida como tal en las clasificaciones científica institucionalizadas" (Acosta, 2010: 1). La multiplicidad de enfoques teóricos que se emplean para el abordaje de los fenómenos comunicativos deja claro este problema ${ }^{2}$.

Según Jean-Michel Berthelot (2001) el desarrollo genético de las disciplinas está condicionado por tres contextos: pragmático, relacionado con intereses prácticos; metodológico, relacionado con la elaboración de procedimientos técnicos y esquemas de pensamiento; y normativo, constituido por el conjunto de debates que acompañan siempre el desarrollo de una disciplina. (Berthelot, 2001, citado en Giménez, 2009: 2). Por lo anterior, hoy la ciencia de la comunicación se presenta como "una disciplina esencialmente híbrida, descentrada, sin fronteras precisas y sin esquemas explicativos propios" (Giménez, 2009: 4). Por eso se afirma que la comunicación se encuentra en una posición de encrucijada de todas las disciplinas sociales: "La posición de encrucijada que ocupa la ciencia de la

2 En un trabajo anterior, se presenta un ensayo en torno a las múltiples formas de organización de las teorias de la comunicación y el impacto que esta multiplicidad tiene en la constitución cognoscitiva del campo de la comunicación. Ver Rizo García (2012a). 
comunicación en el campo de las ciencias sociales debería estimular el diálogo permanente y transdisciplinario entre los comunicólogos y los colegas de las demás disciplinas sociales" (Giménez, 2009: 16).

Como las teorías de la comunicación se han apropiado de paradigmas muy diversos, la comunicación se ve hoy como una especie de archipiélago disperso de disciplinas. Esta particularidad no hay que concebirla esencialmente como algo negativo o perjudicial para el avance del conocimiento en el campo de la comunicación. Para Giménez, por el contrario, la pluralización y la hibridación son más bien signos de vitalidad y de buena salud, con gran "fuerza germinativa" (Giménez, 2009: 5).

Como se puede observar, existe una tendencia bastante generalizada a no considerar a la comunicación como una disciplina autónoma. En muchas ocasiones esta tendencia va acompañada de afirmaciones categóricas en torno a la imposibilidad de la comunicación como disciplina, sin que quede claro lo que se está entendiendo por tal. De ahí que nos parezca pertinente explorar, aunque sea en términos generales, la propia noción de disciplina.

Una disciplina "tiende naturalmente a la autonomía, por la delimitación de sus fronteras, la lengua que ella se constituye, las técnicas que ella está conducida a elaborar o a utilizar, y eventualmente por las teorías que le son propias" (Morin, 1994: 14, citado en Pereira, 2005: 418). También, para que un conjunto de conocimientos se constituyan como disciplina debe haber claridad y precisión en el objeto de estudio, algo que no sucede con la comunicación, por la propia dispersión y multiplicidad de significados que el propio término "comunicación" connota ${ }^{3}$. Si el campo de la comunicación es, por naturaleza, un campo inter y transdisciplinar, es, entre otras razones, porque el propio fenómeno comunicativo es susceptible de ser analizado desde múltiples enfoques, y como ha quedado claro anteriormente, las matrices teóricas desde las cuales se ha investigado la comunicación a lo largo de la historia no pueden agruparse en un único "modo de ver" adscrito a una única disciplina. Es claro, entonces, que "la comunicación no puede ser encasillada en los márgenes de un compartimento disciplinario estanco" (Torrico, 2005: 42).

Entonces, si la comunicación más bien es un campo inter y transdisciplinario, parece poco factible poder hablar de la comunicación como una ciencia, al menos en el sentido clásico del término. Tradicionalmente, para que un conjunto de conocimientos se constituyan como ciencia se requiere la búsqueda de una verdad universal como valor supremo; la verificación empírica o contrastación con el mundo real y natural, y la objetividad. Es obvio que la producción académica que se ha generado a lo largo de la historia del campo académico de la comunicación no cumple con estos requisitos. Por lo anterior, queda claro

3 Un ejemplo de la diversidad de significados del término comunicación lo encontramos en la clasificación que sobre el concepto hace Papalini (2002) con base en la taxonomía de Luciano Gallino (1995). La autora propone los siguientes modos de concebir a la comunicación: a) Como simple transmisión de un estado o propiedad, que puede referirse a objetos inanimados; b) como un comportamiento de un ser viviente que influye sobre otro; c) como intercambio de valores sociales; d) como transmisión de información; e) como el acto de compartir significados socialmente intercambiados; y f) como formación de una unidad social que comparte valores, un determinado modo de vida y un conjunto de reglas. 
que la comunicación está más cercana al concepto de campo, como ya se apuntó en el apartado anterior. Así vista, la comunicación

es un campo de conocimiento desde el cual se puede comprender, interpretar e intervenir a múltiples niveles los procesos de interacción y significación a través de la creación, circulación y usos de medios y tecnología y de formas simbólicas con multiplicidad de perspectivas: social, cultural, ética, política, estética y económica, entre otras (Pereira, 2005: 421).

Con respecto a la noción de transdisciplina, Nicolescu (1999, citado en Múnera, 2010: 15) afirma que ésta "concierne como lo indica el prefijo trans, a lo que simultáneamente es entre las disciplinas, a través de las disciplinas y más allá de toda disciplina. Su finalidad es la comprensión del mundo presente, uno de cuyos imperativos es la unidad del conocimiento". La siguiente afirmación aclara la diferencia entre la inter y la transdisciplina, y permite anticipar, como veremos después, que el campo de la comunicación no alcanza todavia la transdisciplina:

Por analogía con la música, la interdisciplina puede entenderse como polifonía, esto es, como diversidad en la unidad, mientras que la transdisciplina tiene su par en la sinfonía, unidad en la diversidad, es decir, en la pretensión de articulación total, que implica la noción de cierre o clausura (Múnera, 2010: 15).

El fenómeno comunicativo es, sin duda, interdisciplinar, pues sobre él (unidad) se han escrito, investigado, reflexionado e interpretado múltiples cuestiones (diversidad). Prueba de ello es la multiplicidad de perspectivas desde las cuales se ha abordado el fenómeno. La "pretensión de articulación total" a la que debiera aspirar la transdisciplinariedad parece no estar aún lograda en el campo académico de la comunicación, toda vez que no parece haber el consenso necesario ni siquiera en la definición del propio fenómeno comunicativo, polisémico donde los haya y aglutinador de una serie de procesos a los que se da un énfasis distinto según sea la mirada desde la cual lo estemos observando. Lo anterior se sitúa en la línea de lo comentado por otros autores como Migdalia Pineda (2004), para quien

los objetos de estudio de la comunicación se han construido desde miradas múltiples pero en sus primeras aproximaciones se mantuvieron parcelas y es en los últimos años, especialmente desde los ochenta en adelante, que se busca integrar en una visión más interdisciplinaria con un sentido de mayor totalidad, para poder avanzar en la construcción de un pensamiento comunicacional transdisciplinario todavía no consolidado.

Martin Barbero (1992) ahonda en lo anterior, al postular que el necesario avance hacia la transdisciplinariedad en los estudios de la comunicación implica "no significa 
la disolución de sus objetos en los de las disciplinas sociales sino la construcción de las articulaciones - mediaciones e intertextualidades - que hacen su especificidad". En un sentido similar, Vasallo (2002) argumenta que el campo de estudios de la comunicación tiende hacia la transdisciplinariedad, a la que comprende como "un movimiento hacia la superación de los límites entre especialidades cerradas y jerarquizadas y el establecimiento de un campo de discurso y prácticas sociales cuya legitimidad académica y social dependa cada vez más de la profundidad, extensión, pertinencia y solidez de las explicaciones que produzca y no del prestigio institucional acumulado " (Vasallo, 2002: 53).

Desde este punto de vista, la comunicación es, entonces, un campo multidisciplinario $y_{1}$ a lo sumo, interdisciplinario. Al menos en estos momentos. El debate, a veces, roza la paradoja: por un lado, toda actividad humana tiene que ver con la comunicación, y por lo tanto, este fenómeno no puede adscribirse a una única perspectiva teórica ni metodológica; por el otro, el estudio de la comunicación se apoya de otros campos de conocimiento como la lingüística, la semiótica, la sociología, aunque no se trate de un agregado de discursos teóricos que la conviertan automáticamente en un dominio de estudios interdisciplinar. Así entonces, estamos ante un objeto de estudio que por su propia naturaleza debe interesar a varias disciplinas, pero esta misma condición hace que la consolidación teórica del campo de la comunicación siga sin lograrse.

El debate está servido, y es importante transitar de las reflexiones en torno al objeto a las propuestas en torno al sujeto, al campo de conocimiento que mira los fenómenos comunicativos, entrelazados, obviamente, con otras dimensiones de la vida social y cultural. Así, como afirma Torrico, "lejos de optar por la cómoda posición de afirmar que si la Comunicación no llega a ser una ciencia en el sentido convencional no tiene otro camino que convertirse en mera herramienta de la eficacia transmisiva, se requiere más bien pensarla de otro modo" (Torrico, 2005: 42). Para ello usa el concepto de campo científico en el sentido de Pierre Bourdieu y de espacio transfronterizo de Wallerstein... para "dar cuenta de las inherentes multidimensionalidad, transdisciplinariedad y conflictividad que caracterizan a la comunicación como zona de la realidad factible de ser investigada y, por ende, conceptualizada" (Torrico, 2005: 42). Nuevamente, la naturaleza objetual de la comunicación como fenómeno, parece estar al centro.

Otra visión que aboga por la interdisciplinariedad y la transdisciplinariedad del campo de la comunicación es la que propone Alejandro Grimson (2003). Para el autor, la disciplinarización es un proceso de exclusión y de fronterización. Dice que en la disputa sobre la especificidad del campo se debemos "renunciar a la mayor riqueza del campo comunicacional que es, justamente, su carácter inter y transdisciplinario" (Grimson, 2003: 48). Riqueza que, no obstante, no está exenta de conflictos.

En la misma línea, Rodrigo Browne Sartori y Víctor Silva Echeto (2005) afirman que "la comunicación no legitima ningún proyecto epistemológico, su deterritorialización le permite escaparse de los ámbitos cerrados propios de las epistemologías positivistas. De esa forma, se escapa de los preceptos que la pueden encasillar en un código exacto y 
preciso" (Browne y Silva, 2005: 210). Los autores comentan que el hecho de que la comunicación no sea una disciplina ni una ciencia es una ventaja que le permite construir ese tercer espacio, eliminando los territorios cerrados y unidireccionales, para poder salirse del margen ${ }^{4}$. Pero aquí vale la pena preguntarnos: ¿de qué márgenes hablamos? ¿Sin tenerlos claros podemos salirnos de ellos? ¿Qué implicaciones tendría esta superación de los límites para la consolidación del espacio académico de la comunicación?

Atendiendo a lo que afirma Sofía Magdalena Palau (2006),

ante el desdibujamiento de las líneas divisorias entre las disciplinas de las ciencias sociales, algunos académicos interesados en los estudios de comunicación recurrieron a otras dos vías. La primea fue considerar a los estudios de comunicación como un ámbito post-disciplinario, es decir más allá y posterior a la disciplinarización de las ciencias sociales (...) Una segunda vía, tomada por un grupo minoritario de académicos, fue la antidisciplina. Esta posición implica un rompimiento más radical, no sólo con la forma en que se estructuró el campo de las ciencias sociales en términos institucionales y disciplinares, sino como un cuestionamiento al pensamiento científico ortodoxo como única forma de conocimiento válido (Palau, 2006: 36-37).

Según la autora, la primera vía no logró fructificar porque los actores que la propusieron no contaban con suficiente peso específico en la lucha al interior del campo científico de la comunicación.

Las preguntas que se plantearon anteriormente en torno a la propuesta de que la comunicación construya un tercer espacio académico saliéndose de los márgenes estancos de las disciplinas son, sin duda alguna, preocupaciones que se han expresado en muchas ocasiones a lo largo de la historia del campo de la comunicación. Destacamos, por ejemplo, la apreciación de Olmedo: "No sin buenas intenciones, algunos entienden que buscar fronteras temáticas para esta disciplina significa impedir su mayor ventaja, que sería justamente, la de la apertura. Pero es en esta pretendida apertura justamente donde se genera el desorden epistemológico que deriva en la falta de legitimación de nuestro campo. La comunicación termina siendo un campo desdibujado, desde donde se puede analizar casi cualquier problemática, ya que suele entenderse que en definitiva todo comunica y la comunicación es cultura (y viceversa)" (OImedo, 2012: 21-22).

Este desdibujamiento ha hecho que, pese al gran avance en la institucionalización de los estudios sobre comunicación, el campo siga adoleciendo de ciertos obstáculos o lastres que impiden que sea tomado en cuenta como un campo científico. Interdisciplinario, sí, pero científico, también. Algunos de estos lastres, que ya han ido quedando apuntados, son la indefinición del objeto de estudio, la condición tripartita del campo -que si bien puede dar lucidez en términos de organización administrativa del campo, no otorga claridad en

4 Incluso, llevando su posicionamiento al extremo, Browne y Silva afirman que "es necesario pensar desde la no-ciencia, desde la no-disciplina, desde el (in)disciplinamiento que habilita la no-disciplina comunicación" (Browne y Silva, 2005: 219). 
torno a las particularidades del campo de conocimiento en cuestión - y la dependencia de la comunicación de otros campos del saber con mayor trayectoria histórica y mayor legitimidad científica, tales como la sociología y la antropología.

\section{Miradas disciplinarias: las posibilidades de la comunicación como ciencia}

El campo de la comunicación, cuyos orígenes se remontan a la primera mitad del siglo XX, siguió los movimientos generalizados de las ciencias sociales de ese entonces por conseguir un estatuto científico particular. Esfuerzos como los de Wilbur Schramm (1982), que trató de "reunir un cuerpo de conocimientos teóricos, un grupo de objetos de estudio específicos y una orientación metodológica son clara evidencia de ese impulso por alcanzar el estatus disciplinar para los estudios de comunicación" (Palau, 2006: 35).

Si bien como ha quedado claro, parece existir una opinión generalizada en torno a que la comunicación no constituye una disciplina, sino un campo de conocimiento interdisciplinario (que va dando pequeños pasos hacia la transdisciplinariedad), existen algunas propuestas que sí abogan por una mirada disciplinaria al campo. En este sentido, vale la pena recuperar, al menos, dos miradas: la de Jesús Galindo y los trabajos realizados por el Grupo hacia una Comunicología Posible durante el periodo 2003-2009, por un lado, y las críticas expresadas por Roberto Follari en torno a la dificultad de hablar de un campo interdisciplinario que aún no ha logrado su constitución disciplinaria, por el otro.

El Grupo hacia una Comunicología Posible (GUCOM), nacido en México en el año 2003, partió de la necesidad de fundamentar científicamente a la Comunicación. El argumento, en ese entonces, fue claro: era necesario contar con un piso teórico común en el que poder aterrizar los diversos proyectos de investigación que en aquellos momentos se estaban desarrollando en el incipiente Doctorado en Comunicación de la Universidad Veracruzana (México), coordinado por Jesús Galindo Cáceres, quien sería el impulsor de los trabajos del GUCOM durante los casi siete años de trabajo ininterrumpido del grupo. La construcción de ese piso teórico común debía partir de la revisión de las propuestas de clasificación de las teorías de la comunicación existentes en la literatura académica sobre el tema. Aquí cabe señalar que el trabajo del GUCOM, en un primer momento, fue de corte documental, esto es, se realizó investigación bibliográfica en aras de identificar las principales corrientes teóricas que permitían hablar de la existencia de un pensamiento comunicacional con cerca de un siglo de existencia ${ }^{5}$.

5 Las propuestas que fueron punto de arranque para la propuesta de las fuentes científicas históricas de la comunicología que plantearia el GUCOM unos años después fueron la de Lazar (1995), Miege (1996), Mattelart (1997) y Torrico (2004). Una propuesta que mereció una revisión más detallada fue la planteada por Robert Craig (1999), que incluia siete tradiciones básicas de los estudios de comunicación que daban lugar, a su vez, a siete formas distintas de concebir el fenómeno comunicativo. Las tradiciones establecidas por Craig son las siguientes: tradición retórica (la comunicación como el arte práctico del discurso); tradición semiótica (la comunicación como la mediación intersubjetiva por medio de signos); tradición fenomenológica (la comunicación como la experiencia del uno y del otro, o de los otros, por medio del diálogo); 
A lo largo de sus años de existencia, el GUCOM trabajó en tres frentes distintos: uno bibliográfico-hemerográfico ${ }^{6}$, uno historiográfico y uno de construcción teórica-epistemológica. La primera etapa de trabajos incluyó la revisión de la bibliografía básica escrita en español sobre comunicación y tuvo como propósito identificar los principales objetos de estudio, enfoques y tendencias de la producción académica en comunicación, con énfasis en México y América Latina, pero también contemplando la literatura fundamental extranjera con traducción a la lengua española.

El esquema de trabajo partió de la entonces propuesta incipiente de la Comunicología propuesta por Jesús Galindo en el año 2003-2004, que incluía cuatro dimensiones comunicológicas básicas (expresión, difusión, interacción y estructuración) ${ }^{7}$ y la consideración de las siete fuentes científicas históricas de la comunicología (sociología funcionalista, sociología crítica-cultural, sociología fenomenológica, psicología social, economía política, semio-lingüística y cibernética) ${ }^{8}$.

El segundo proyecto de trabajo del grupo fue de corte historiográfico. La tarea de reconstrucción histórica del pensamiento comunicacional implicó trabajar desde la óptica de la historia de la ciencia. La tarea consistió en desarrollar las genealogías de las nueve fuentes científicas históricas de la comunicología, desde sus orígenes hasta su impacto y presencia en el pensamiento en comunicación. Este segundo frente de trabajo tuvo como producto tres libros colectivos, que contaron con la participación de todos los miembros del grupo 9 .

El tercer y último frente de trabajo del GUCOM fue el teórico-epistemológico. En este tenor, y a partir de lo abordado en los proyectos anteriores las preguntas y planteamientos del grupo se dirigieron hacia la propuesta concreta de construcción de la ciencia de la comunicación, la Comunicología. En este punto, se advirtió que no era posible hablar de una única Comunicología, ya que dependiendo del enfoque y de la fuente científica histórica a la cual se le otorgara mayor importancia, se podian proponer distintas comunicologías, nueve en total. De ahí que la obra producto de esta etapa de trabajo incluya las nueve propuestas de construcción de una ciencia general de la comunicación, una por cada una de las fuentes científicas del pensamiento comunicacional ${ }^{10}$.

\footnotetext{
tradición cibernética (la comunicación como el procesamiento y la transmisión de información); tradición sociopsicológica (la comunicación como proceso de expresión, interacción e influencia; tradición sociocultural (la comunicación como un proceso simbólico, por medio del cual la realidad es producida, mantenida, recuperada y transformada); y tradición critica (la comunicación como causante de las formas materiales e ideológicas que impiden o distorsionan la reflexión crítica). 6 Ver Galindo, Karam y Rizo (2005).

7 En el campo académico se han atendido fundamentalmente objetos de estudio que tienen que ver con la difusión (los sistemas de información, los medios) y la estructuración (la relación entre la comunicación y otras dimensiones de la vida social, tales como la política, la educación y la cultura, por citar algunas); la expresión (el resultado de la puesta en común con base en los sistemas de códigos -lenguajes - compartidos) y la interacción (la que retoma la acepción originaria del término comunicación como puesta en común, vínculo e intercambio) han sido escasamente estudiadas. Cabe mencionar que posteriormente, hacia el año 2007, la propuesta de GUCOM incluyó una quinta dimensión, la observación, la más compleja de las dimensiones comunicológicas.

8 Posteriormente se dividió la sociología crítica-cultural en dos fuentes, sociología crítica y sociología cultural; y la semio-lingüistica también en dos fuentes, la semiótica y la lingüística. Por ello es que se trabajó posteriormente a partir de nueve fuentes científicas históricas.

9 Ver Galindo (2008), Galindo y Rizo (2008) y Galindo (2010).

10 Ver Galindo, Karam y Rizo (2011).
} 
Los trabajos del Grupo hacia una Comunicología Posible son, a nuestro entender, un buen ejemplo de esta tendencia de opinión que apunta hacia la necesidad de la consolidación y legitimación científica de la comunicación como campo científico particular. Propuesta que, no obstante, no es contradictoria a cabalidad con las lecturas interdisciplinarias, toda vez que incluye miradas propias de otras disciplinas para comprender, por un lado, las aportaciones de éstas al pensamiento comunicacional y, por el otro, la especificidad necesariamente multidimensional de la comunicación como mirada sobre lo social.

Aunque ha habido varios intentos, desde distintos frentes, por considerar a la comunicación como una disciplina, y aunque estos intentos se enmarcan en un contexto de notable crecimiento del campo académico, la comunicación no ha alcanzado la madurez y estabilidad de otras disciplinas científicas. Ello se debe, en parte, a la insuficiente fundamentación teórica de la comunicación. Además, la teoría de la comunicación debe librarse de dos viejos lastres (Moreno, 2008): la indefinición de su objeto de estudio -"todo es comunicación" - y la identificación con la comunicación de masas. Otra forma de expresar este problema lo encontramos en la siguiente afirmación de Jesús Galindo (2005: 17): "tenemos comunicólogos pero no tenemos Comunicología". Lo anterior se inserta en el debate en torno a la distinción de la comunicación como campo educativo y profesional, por un lado, y como campo de conocimiento científico, por el otro. Así entonces, parte del problema de la indefinición de la comunicación como campo científico es producto, también, de que la comunicación surgió primero de la profesión para luego trasladarse problemáticamente al campo de lo científico.

Lo anterior va acompañado de la escasa claridad en la delimitación del objeto de estudio de la comunicación, una indefinición que si bien no es reciente, sí es vigente (Lobo, 2007). "Si el campo comunicacional no crea su propio objeto y método, su propia epistemología, estará destinado a la marginación institucional" (Olmedo, 2007: 3). En la misma línea, Torrico (2004) afirma que "la de la comunicación es un área particularmente afectada por ese síndrome de lo light, esa vacuidad, debido en especial al tipo de demanda coyuntural comercial". Para el autor, el objeto de la comunicación es "el proceso social de producción, circulación mediada, intercambio desigual, intelección y uso de significaciones y sentidos culturalmente situados" (Torrico, 2004).

Otra propuesta que se aleja de las concepciones más comunes de la comunicación como campo interdisciplinario es la de Roberto Follari. Para el autor, los intentos de hacer la comunicación, de facto, un campo interdisciplinario, acarrea problemas epistemológicos graves. Problemas que contribuyen a acrecentar el desdibujamiento y la indeterminación: "La idea de que todo significa, y que ello llevaría a considerar a todo objeto como objeto de los estudios en Comunicación, colabora a la indeterminación y la confusión en académicos y estudiantes, y al consiguiente desprestigio del campo ante otras de las disciplinas sociales" (Follari, 2007).

Desde ese lugar, Follari critica las posturas que plantean la postdisciplinariedad. Para el autor, "no se pueden traspasar las barreras disciplinarias si previamente esas barreras 
no fueron delimitadas, por lo cual propone especificar primero el objeto de la comunicología (Follari, 2001, citado en Sarale, 2008: 6). Aunque ya se comentó que parece no haber mucho problema para lograr un consenso en torno a que la comunicación debe estudiar, primordialmente, los procesos de producción de sentido, esta afirmación es quizás demasiado amplia y no permite concretar un objeto específico tal y como sugiere Follari.

\section{Preguntas para el debate}

La comunicación adolece de varios problemas que merecen atención urgente. Como objeto de estudio, parece bastante claro y consensuado el que la comunicación puede y debe ser abordada desde enfoques disciplinarios muy variados. Como campo profesional existe también cierta claridad en torno a las actividades que realizan los comunicadores en los cada vez más diversificados espacios de desarrollo laboral. Como campo educativo, son cada vez más las ofertas de licenciaturas relacionadas con la comunicación, aunque en esta oferta vemos reflejada, en cierto modo, la confusión en torno al propio término comunicación, pues son muchas las formas de nombrar los estudios en comunicación y muchos y muy diversos los imaginarios con que los estudiantes acceden a este tipo de estudios $^{11}$. Como campo académico y científico, la comunicación sigue siendo marginal con respecto a las ciencias sociales, por un lado, y a las ciencias en general, por el otro; aunque ciertamente ha habido muchos avances en cuanto a su institucionalización, asuntos que puede verse en, por ejemplo, el incremento de la oferta de posgrados en el área, el crecimiento y consolidación de las publicaciones académicas y el papel cada vez mayor de las asociaciones de investigadores como agentes de consolidación de la investigación en torno a los fenómenos comunicativos.

Reconocer los caminos andados permite apuntalar algunas cuestiones que es importante considerar para seguir avanzando como campo académico y científico. Si bien no se puede negar la naturaleza interdisciplinaria del fenómeno comunicativo, asi como la diversidad de enfoques que convergen en el campo académico de la comunicación, es importante poner al centro el debate en torno a la necesidad de fortalecer teórica y epistemológicamente el conocimiento que en el campo de la comunicación se produce.

El reconocimiento de la historia del pensamiento comunicacional da cuenta de la diversidad de enfoques, tendencias, acercamientos y aproximaciones que ha habido sobre el fenómeno de la comunicación a lo largo de más de cien años de existencia institucional de los estudios de comunicación. Ello parece claro. Sin embargo, no es tan clara la definición de los objetos de estudio que son propios del campo científico de la comunicación, y por ende, tampoco es tan clara la particularidad del campo. Como se ha manifestado a lo largo

11 En una investigación anterior, se ofrecen datos relevantes en torno a los imaginarios sobre la comunicación de estudiantes de licenciatura en México. Ver Rizo (2012b). 
del presente texto, son varios los polos de tensión en el debate en torno al estatuto de la comunicación como campo científico: un primer polo está constituido por la dicotomía entre pensar que existe una teoría general de la comunicación o, por el contrario, concebir que el fenómeno comunicativo necesariamente no puede investigarse en el paraguas de una única propuesta o mirada teórica; un segundo polo, que aglutina los problemas tanto estructurales como educativos y profesionales, tiene que ver con la dicotomía entre la teoría y la práctica de la comunicación; en tercer lugar, las tensiones sobre el objeto de la comunicación parecen, al menos, sostenerse en un consenso básico que apunta a que la comunicación no sólo debe investigar los procesos mediáticos, sino las mediaciones, los procesos de construcción de sentido. (Lobo, 2007).

Tomando en cuenta que, sin duda alguna, la comunicación puede ser concebida como un campo joven "en permanente problematización" (Lobo, 2007), es natural que sean muchas las voces académicas que están preocupadas por abonar elementos para seguir discutiendo esta problemática. "Concebida como una disciplina reciente, la comunicación, lejos de haber esclerosado su objeto de estudio, delimitando con precisión sus fronteras, se encuentra en el camino de la superación de los obstáculos epistemológicos que supuso el estudio de los medios" (Lobo, 2007).

No tener claro el objeto es, sin duda, un gran obstáculo para la consolidación de la comunicación como campo científico. Aunque quizás sea esta ausencia de un solo objeto claro lo que defina a este campo de conocimiento que, necesariamente, debe interactuar con otros enfoques y disciplinas en aras de construir conocimiento sobre las realidades sociales actuales, mismas que no pueden comprenderse sin atender lo comunicativo.

"Si pensamos que la ciencia es en definitiva una cuestión histórico-cultural y de poderes también, las definiciones al interior del campo de la comunicación no se deberán pretender como dogmáticas y únicas y aceptar este juego que provoca los desplazamientos del objeto de estudio de la comunicación en eras de pensar un camino de consolidación del campo" (Lobo, 2007).

Coincidimos en la necesidad de apertura de miras. El dogmatismo puede ser peligroso, e incluso contraproducente, en un campo de conocimiento que lucha por hacerse un lugar en el campo mayor del pensamiento y la investigación social: "el diálogo académico debe procurarse independientemente si se llega al consenso o al disenso. Desde la diferencia también se construye" (Echeverry, 2009: 53).

Aunque existen autores que consideran que la reflexión epistemológica al interior del campo de la comunicación no es suficiente -Tiago Quiroga, por ejemplo, considera que uno de los obstáculos para la consolidación de la comunicación es la "escasez de las propias reflexiones epistemológicas en el campo" (Quiroga, 2013: 37) -, la propia discusión en torno a los múltiples posicionamientos que existen sobre el estatuto epistemológico de la comunicación da cuenta de un campo de conocimiento de enorme vitalidad y dinamismo, que se mira a sí mismo y que trata de ir logrando cada vez más consensos al interior de la comunidad académica. Este "tratamiento autoreflexivo de la comunicación académica" 
(Echeverry, 2009: 7) habla, sin lugar a dudas, de un dominio de estudios central en sociedades como las actuales, incomprensibles sin tomar en cuenta las múltiples formas de comunicación y los efectos socioculturales derivados de ellas.

\section{Referencias}

ACOSTA, Ángel (2010). La comunicación, ¿bisagra epistemológica?. Ponencia presentada en el II Congreso de la Asociación Española de Investigación de la Comunicación (AE-IC), Universidad de Málaga (España), 3, 4 y 5 de febrero de 2010. Dirección: http://www. aeic2010malaga.org/upload/ok/363.pdf (Última consulta: 19 de octubre de 2013).

BERTHELOT, Jean-Michel (2001). Épistemologie des sciences sociales, Paris : Presses Universitaires de France.

BOURDIEU, Pierre (1997). El oficio de sociólogo, México: Siglo XXI.

BROWNE SARTORI, Rodrigo y SILVA ECHETO, Victor (2005). Las indisciplinas de la comunicación. Epistemologías en crisis, en Comunicación, Núm. 3 (2005), pp. 209-220, Departamento de Comunicación Audiovisual y Publicidad y Literatura, Sevilla: Universidad de Sevilla. Dirección: http://www.revistacomunicacion.org/pdf/n3/articulos/ las_disciplinas_de_la_comunicacion_epistemologias_en_crisis.pdf (Última consulta: 10 de noviembre de 2013).

CORNEJO, Hugo (2007). Tránsitos de las ciencias sociales a las ciencias de la comunicación, en Tecsistecatl, Vol. 1, Núm. 1, Enero 2007. Dirección: http://www.eumed.net/rev/ tecsistecatl/n0/hcmc1.pdf (Última consulta: 9 de octubre de 2013).

DOGAN, Mattei y PHARE, Robert (1991). L'innovation dans les sciences sociales, Paris: Presses Universitaires de France.

ECHEVERRY MEJÍA, Jorge Andrés (2009). Epistemología de la comunicación en lberoamérica. Contribución al estudio de la comunicación, Monografía. Facultad de Comunicaciones, Pregrado en Comunicaciones, Universidad de Antioquia, Medellín: Universidad de Antioquia. Dirección: http://comunicaciones.udea.edu.co/autoevaluacioncomunicaciones/images/Sitio_informe/Anexos/Otros\%20Informes/Monograf\%C3\%ADa_Jorge_Andr\%C3\%A9s/Monografia_Jorge_Andres_EM.pdf (Última consulta: 28 de octubre de 2013).

FOLLARI, Roberto (2001). Relevo de las ciencias sociales latinoamericanas. Estudios culturales, transdisciplinariedad y multidisciplinariedad, en Revista Diálogos de la Comunicación, Núm. 63, Lima: FELAFACS.

FOLLARI, Roberto (2003). La moldura en espejo. Encrucijadas epistemológicas de las ciencias de la comunicación, en Tram(p)as de la comunicación y la cultura, Núm. 16, La Plata (Argentina).

FOLLARI, Roberto (2007). La formación imposible. Ponencia presentada en las XI Jornadas 
de Investigadores en Comunicación: tramas de la comunicación en América Latina contemporánea. Tensiones sociales, políticas y económicas. Mendoza (Argentina), 4, 5 y 6 de octubre de 2007.

FUENTES NAVARRO, Raúl (1999). La investigación de la comunicación en América Latina: condiciones y perspectivas para el siglo XXI, en Revista Diálogos de la Comunicación, Núm. 56, octubre 1999, Lima: FELAFACS.

FUENTES NAVARRO, Raúl (2003). La producción social de sentido sobre la producción social de sentido: hacia la construcción de un marco epistemológico para los estudios de la comunicación, en Vassallo de Lopes, Maria Immacolata (org.) Epistemologia da Comunicação. São Paulo: Loyola (Comunicação Contemporânea No. 1), pp.15-40.

GALINDO, Jesús (2005). Sobre comunicología y comunicometodología. Primera guía de apuntes sobre horizontes de lo posible, en Culturales, Enero-Junio, año/vol.1, núm. 001, Universidad Autónoma de Baja California, México, pp. 7-28.

GALINDO, Jesús; KARAM, Tanius y RIZO, Marta (2005). Cien libros hacia uma comunicología posible, México: Universidad Autónoma de la Ciudad de México.

GALINDO, Jesús (coord.) (2008). Comunicación, ciencia e historia. Fuentes científicas históricas hacia una comunicología posible, Madrid: McGraw Hill Interamericana.

GALINDO, Jesús y RIZO, Marta (coords.) (2008). Historia de la comunicología posible. Las fuentes de un pensamiento científico en construcción, León y Puebla: Universidad Iberoamericana León y Universidad Iberoamericana Puebla.

GALINDO, Jesús (2010). Sociología y Comunicología. Historias y posibilidades, Salta (Argentina): Universidad Católica de Salta.

GALINDO, Jesús (2011). Comunicología posible. Hacia una ciencia de la comunicación, México: Universidad Intercontinental.

GALLINO, Luciano (1995). Diccionario de sociología, México: Siglo XXI.

GIMÉNEZ, Gilberto (2009). Comunicación, cultura e identidad. Reflexiones epistemológicas. Ponencia presentada el 22 de abril de 2009 en el IV Coloquio Internacional de Cibercultur@y Comunidades Emergentes de Conocimiento local: Discurso y Representaciones Sociales, organizado por el Laboratorio de Comunicación Compleja, Centro de Estudios e Investigaciones Interdisciplinarias en Ciencias y Humanidades, UNAM, y el Colegio de San Luis Potosí. San Luis Potosí. Dirección: http://perio.unlp. edu.ar/teorias2/textos/m4/gimenez.pdf (Última consulta: 2 de noviembre de 2013).

GIMÉNEZ, Gilberto (2012). Pluralidad y unidad de las ciencias sociales. Ponencia presentada en el XVII Congreso Nacional de Posgrado, UNAM, Coordinación de Estudios de Posgrado, pp. 249-261. Dirección: http://www.posgrado.unam.mx/publicaciones/ ant_omnia/Esp_xvii_cong/29.pdf (Última consulta: 2 de noviembre de 2013).

GRIMSON, Alejandro (2003). Contra una epistemología de la comunicación. Una crítica al corporativismo lógico, en Revista Zigurat, Núm. 4, Facultad de Ciencias Sociales, Universidad de Buenos Aires, pp. 43-48.

HERCOVICl, Alain (1998). El estatuto epistemológico de las ciencias de la comunicación, 
en Causas y Azares, Núm. 7, pp. 131-141, Buenos Aires. Dirección: http://ateneodecomunicacion.files.word press.com/2012/08/el-estatuto-epistemolc3b3gico-de-lasciencias-de-la-comunicacic3b3n-por-alain-herscovici.pdf (Última consulta: 27 de octubre de 2013).

KARAM, Tanius (2007). Epistemología y comunicación. Notas para un debate, en Andamios. Revista de investigación social, Vol. 4, Núm. 7, Diciembre, Colegio de Humanidades y Ciencias Sociales, Universidad Autónoma de la Ciudad de México, pp. 97-124.

LAZAR, Judith (1995). ¿Qué sé? La ciencia de la comunicación, México: Publicaciones Cruz. LEÓN DUARTE, Gustavo A. (2009). Estrategias, posiciones y prácticas científicas en la enseñanza y la investigación de la comunicación en América Latina. Ponencia presentada en el XIII Encuentro Latinoamericano de Facultades de Comunicación Social (FELAFACS), La Habana (Cuba), 19-22 de octubre de 2009.

LOBO, Claudio Tomás (2007). Definiciones para un objeto de estudio de la comunicación. Tensiones latinoamericanas. Ponencia presentada en las XI Jornadas Nacionales de Investigadores en Comunicación, Universidad Nacional de Cuyo, Mendoza (Argentina). Dirección: http://www.redcomunicacion.org/memorias/pdf/2007Lilobo.pdf (Última consulta: 2 de noviembre de 2013).

MARTÍN BARBERO, Jesús (1992). Pensar la sociedad desde la comunicación. Un lugar estratégico para el debate de la modernidad. En revista Diálogos de la comunicación, Núm. 32, FELAFACS, Lima (Perú). Dirección: http://www.dialogosfelafacs.net/revista/ upload/articulos/pdf/32JesusMartin.pdf (Última consulta: 5 de marzo de 2014).

MARTÍN SERRANO, Manuel (1986). Presentación de la teoría social de la comunicación, en Revista Española de Investigaciones Sociológicas, Núm. 33, Centro de Investigaciones Sociológicas, Madrid, pp. 15-34. Dirección: http://www.reis.cis.es/REIS/PDF/ REIS_033_04.pdf (Última consulta: 23 de octubre de 2013).

MARTINO, L. C. (2002). Elementos para una epistemología de la comunicación, en Vasallo de Lopes, M. Immacolata y Fuentes Navarro, Raúl (comps.) (2002). Comunicación, campo y objeto de estudio, Guadalajara: ITESO.

MATTELART, Armand (1997). Historia de las teorías de la comunicación, Barcelona: Paidós. MAUSS, Marcel (1979). Ensayo sobre los dones: motivo y forma del cambio en las sociedades primitivas, en Sociología y Antropología: 155-268. Madrid: Tecnos.

MIĖGE, Bernard (1996). El pensamiento comunicacional, Mexico: Universidad Iberoamericana.

MORIN, Edgar (1994). Sobre la interdisciplinariedad. Contribución al Congreso de d'Arrabida, Bulletin Interactif du Centre Internationale de Recherches et Études Transdisciplinaires, 2.

MÚNERA, Pablo (2010). Una aproximación in-disciplinaria a la epistemología de la comunicación. En Encuentros, Vol. 8, Núm. 15. Universidad Autónoma del Caribe. Barranquilla (Colombia), pp. 11-23. Dirección: http://www.uac.edu.co/images/stories/ publicaciones/revistas_cientificas/encuentros/volumen-8-no-15/art01.pdf (Última 
consulta: 5 de marzo de 2014).

NICOLESCU, B. (1999). La transdisciplinariedad. Manifiesto de París. París: Ed. Du Rocher. OLMEDO, Gisela (2007). Interrogantes acerca del estatuto epistemológico de la comunicación. Acercamiento a caminos propuestos. Ponencia presentada en las XI Jornadas Nacionales de Investigadores de la Comunicación, UNCUYO, Mendoza. Dirección: http://redcomunicacion.org/memorias/pdf/2007Giolmedo.pdf (Última consulta: 5 de marzo de 2014).

OLMEDO SOSA, Gisela (2012). Estatuto epistemológico de las teorias que abordan la Comunicación Social (esbozo sobre la peculiaridad que ellas presentan en América Latina), en Algarrobo, Año 1, Núm. 1, Revista on-line de la Maestría en Estudios Latinoamericanos, Facultad de Ciencias Políticas y Sociales, Universidad Nacional de Cuyo, Mendoza (Argentina). Dirección: http://revistas.uncu.edu.ar/ojs/index.php/mel/ article/view/2/6 (Última consulta: 4 de noviembre de 2013).

OROZCO, Guillermo (1994). Comunicadores hacia el año 2000: desafíos pedagógicos de su formación, en Diálogos de la comunicación, Núm. 39, Lima: FELAFACS, pp.78-89.

PALAU CARDONA, Magdalena Sofía (2006). El campo de la comunicación: algunas discusiones abiertas, en Rebeil Corella, María Antonieta, XIII Anuario de Investigación de la Comunicación, México: Universidad Anáhuac/ Universidad lberoamericana, pp. 31-50.

PAPALINI, Vanina A. (2002). La comunicación: espacio teórico para la ideología contemporánea y su crítica. Ponencia presentada en el VI Congreso Latinoamericano de Investigadores de la Comunicación, Santa Cruz de la Sierra (Bolivia), junio de 2002. Documento mimeografiado.

PEREIRA, José Miguel (2005). La comunicación: un campo de conocimiento en construcción. Reflexiones sobre la comunicación social en Colombia, en Investigación y Desarrollo, Vol. 13, Núm. 2 (2005), Bogotá: Universidad del Norte. Dirección: http:// redalyc.uaemex.mx/src/inicio/ArtPdfRed.jsp?iCve=26813209 (Última consulta: 10 de noviembre de 2013).

PINEDA, Migdalia (2004). La investigación de la comunicación en América Latina: ¿cómo lo hacemos y hacia dónde vamos?. Ponencia presentada en el VII Congreso de ALAIC. Documento mimeografiado.

QUIROGA, Tiago (2013). Sobre o campo científico da comunicação, en Questões Transversais - Revista de Epistemologias da Comunicação, Vol. 1, Núm. 1, Enero-Julio 2013, São Leopoldo: UNISINOS. Dirección: http://revistas.unisinos.br/index.php/questoes/ article/view/5712/pdf (Última consulta: 10 de noviembre de 2013).

RIZO GARCÍA, Marta (2012a). Interdisciplina y dispersión en las teorías de la comunicación. Apuntes sobre la constitución cognoscitiva del campo de la comunicación y la enseñanza de las teorias de la comunicación, en Castellanos Cerda, Vicente; PÉREZ SALAZAR, Gabriel y AGUILAR EDWARDS, Andrea (eds.) (2012) La producción social del conocimiento en las ciencias de la comunicación y su incidencia social, México: Universidad Autónoma de Coahuila y AMIC. 
RIZO GARCÍA, Marta (2012b) Imaginarios sobre la comunicación. Algunas certezas y muchas incertidumbres en torno a los estudios de comunicación, hoy. Bellaterra (Barcelona, España): Institut de la Comunicació, Universitat Autònoma de Barcelona. Dirección: http://incom.uab.cat/download/eBook_2_InComUAB_MRizo.pdf (Última consulta: 10 de noviembre de 2013).

SÁNCHEZ RUIZ, Enrique E. (1997) Algunos retos para la investigación mexicana de comunicación. Una reflexión personal (en diálogo con Raúl Fuentes). En Comunicación y Sociedad, núm. 30, mayo-agosto. DECS-Universidad de Guadalajara. Guadalajara. SARALE, Nicolás (2008). Comunicación, cultura, estudios culturales... La (in)definición del objeto de estudio de la comunicación y de su estatuto (in/inter/multi/trans/post) disciplinario, en Question. Revista especializada en Periodismo y Comunicación, Vol. 1, Núm. 20. Universidad Nacional de La Plata (Argentina). Dirección: http://www. perio.unlp.edu.ar/ojs/index.php/question/article/view/676/581 (Última consulta: 2 de noviembre de 2013).

SCHRAMM, Wilbur (1982). La ciencia de la comunicación humana, México: Grijalbo.

SIERRA, Luis I. (2005) Una aproximación trans e interdisciplinaria del campo de la comunicación. En revista Conexão - Comunicação e Cultura, UCS, Caxias do Sul (Brasil), Vol. 4, Núm. 8, pp. 81-100, Julio-diciembre 2005. Dirección: http://www.ucs.br/etc/revistas/ index.php/conexao/article/viewFile/106/97 (Última consulta: 5 de marzo de 2014).

TORRICO VILLANUEVA, Erick (2004). Abordajes y periodos de la teoría de la comunicación, Buenos Aires: Norma.

TORRICO, Erick (2005). Acercamiento a la comunicación como cultura académica y a sus proposiciones teóricas generales, en Conexão - Comunicação e Cultura, UCS, Caxias do Sul, Vol. 4, Núm. 8, julio-diciembre 2005, pp. 39-49. Dirección: http://www.ucs. br/etc/revistas/index.php/conexao/article/viewFile/101/92 (Última consulta: 3 de noviembre de 2013).

VASALLO DE LOPES, María Immacolata (2002). Reflexiones sobre el estatuto disciplinario del campo de la comunicación, en Vasallo de Lopes, María Immacolata y Fuentes $\mathrm{Na}$ varro, Raúl (comps.) (2002). Comunicación: campo y objeto de estudio. Perspectivas reflexivas latinoamericanas, Guadalajara: ITESO/UAA/UCOL/UDG.

WALLERSTEIN, Immanuel (1999). El legado de la sociología, la promesa de la ciencia social, Caracas: Nueva Sociedad. 